\title{
Research Progress of solar cells with natural dyes constructed as sensitizer
}

\author{
Li Lan ${ }^{1, a}$, Zhao Kunyu ${ }^{2, b}$ and Zhang Yuehua ${ }^{3, c_{*}}$ \\ ${ }^{1}$ College of Science, Jiamusi University, Jiamusi, Heilongjiang province, China \\ ${ }^{2}$ Academy of Environmental Sciences. Jiamusi EPA, Jiamusi, Heilongjiang province, China \\ ${ }^{3}$ College of Science, Jiamusi University, Jiamusi, Heilongjiang province, China

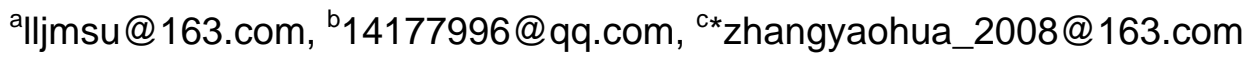

Keywords: Vegetable dyes, sensitizers, conversion, stability, solar energy

Abstract: Solar energy as a renewable energy source has the advantages unmatched by other energy sources. It is inexhaustible, and widely distributed, low cost, safe and will not cause any pollution to the environment. Convert solar energy is an important form of utilization of solar energy in the past ten years, the use of semiconductor photo electrochemical cell instead of the conventional solid-state photovoltaic semiconductor solar cell to complete the conversion of solar energy potential economic value increasingly apparent in many of the semiconductor material, $\mathrm{TiO}_{2}$ to its unique low and stable, widely used. Radiation to the Earth's surface in sunlight, ultraviolet light (4\%), visible accounted for $43 \%$, N-type semiconductor $\mathrm{TiO}_{2}$ band gap of $3.2 \mathrm{eV}$, located absorb the ultraviolet region, the absorption of visible light is weak, in order to increase the use of sunlight rate, people dye adsorbed on the $\mathrm{TiO}_{2}$ surface, with the effect of visible light-sensitive dye, increasing the entire dye-sensitized solar cell for sunlight absorption rate, thus constructed a dye-sensitized solar cell-DSSC (dye-sensitizedsolar-cell) battery .

\section{Introduction}

The energy problem is an important problem restricting the development of world economy, the negative effects of conventional energy sources has been a consensus in the global scope, such as common problems such as environmental pollution, global warming. On the one hand, with the development of economy, conventional energy reserves dwindling; On the other hand the uses of conventional energy sources also produce serious pollution to the ecological environment, leading to increasingly severe environmental conditions. Therefore, it is very urgent to develop new energy task. In 1991, in Switzerland, he considered Michael M.G pretzel [1, 2], a professor at a team of researchers led by using in nano-crystalline $\mathrm{TiO}_{2}$ thin film, carboxylic acids and pyridine ruthenium complexes as dye, making the dye sensitized nano-crystalline solar Cells (Nona-Crystalline Photostatic Cells) components, with $7.1 \%-7.1 \%$ of the photoelectric conversion efficiency, since the dye sensitization solar energy battery has become the countries racing research topic. In 1998, M.G ratzel professor and others through the use of the DSSC structure component, the photoelectric conversion efficiency reached a new high of $10 \%$ to $10 \%$. However, because of the dye sensitized nano-crystalline solar cells using liquid electrolyte, so the emergence of the inevitable influence such as solvent leakage, easy to volatilize battery components many problems, such as stability, thus for encapsulation and light-electric battery, use and store more strict requirements are put forward. To solve these problems, the researchers put forward using quasi solid or solid electrolyte preparation of dye sensitized nanocrystalline solar cells, a new train of thought, so choose based on $\mathrm{TiO}_{2}$ nano-crystalline semiconductor thin film, more than can be ideal to solve many problems. Higher conversion efficiency of the liquid electrolyte, but easy sensitizing dye desorption, sealing difficulties and other problems. The solid electrolyte can avoid these shortcomings, so the development of a high conversion efficiency of the solid electrolyte is important. 


\section{Structure and Work Mechanism of the DSSC}

\subsection{The Basic Structure of DSSC}

DSSC is dominated by the conductive metal oxide, nano semiconductor $\mathrm{TiO}_{2}$ porous films, Dye sensitizer (Dye), electrolytes, and electrode [3] for five parts, as shown in figure 1. The structure of the DSSC is similar to the layers of voltage.

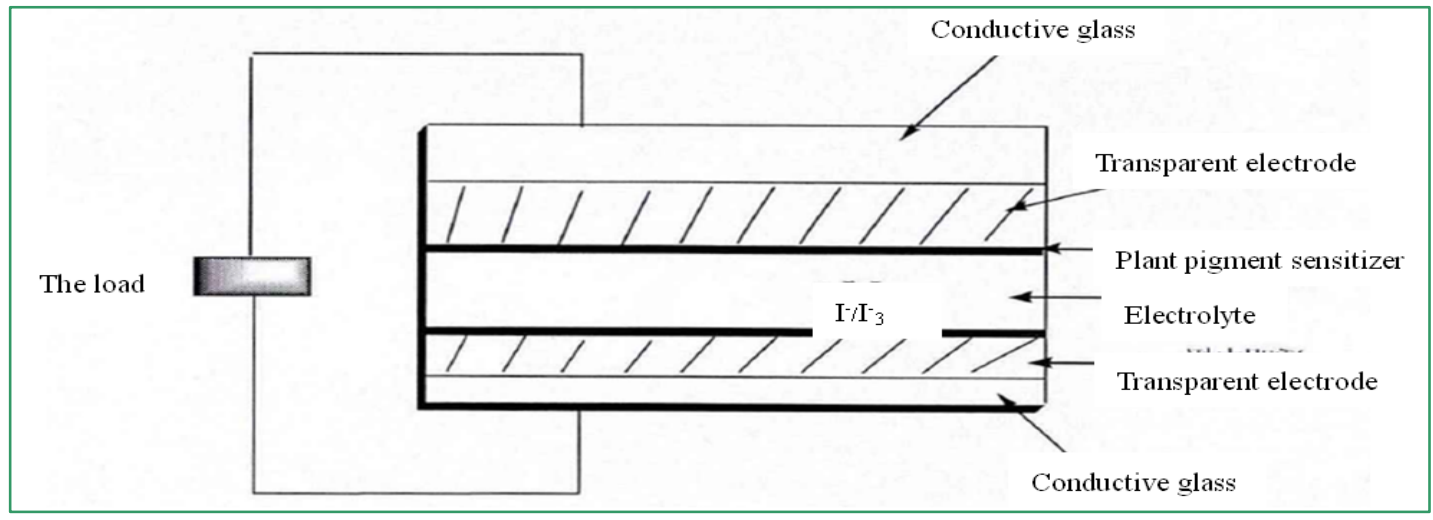

Fig1. The Schematic structure of DSSC

\subsection{The Principle of the DSSC}

Nanocrystalline $\mathrm{TiO}_{2}$ semiconductor thin film, for example, when parallel incident light irradiation to nanocrystalline $\mathrm{TiO}_{2}$ thin film electrode, adsorption in the dye molecules on the surface of the thin film electrode (S) to absorb energy, their electrons are inspired by the ground state transition to the excited states $\left(\mathrm{S}^{*}\right)$ (available formulas (1), the same below), excited electronic quickly intorelatively close $\mathrm{TiO}_{2}$ in the conduction band, dye molecules also loses electrons (e - (CB)) and into oxidation state (2) into the $\mathrm{TiO}_{2}$ conduction band of electron transport in nanocrystalline $\mathrm{TiO}_{2}$ semiconductor thin film and enrichment to the conductive metal oxides, which make the electron accumulation on conductive glass, and then by the external flow of electrode; In the oxidation state of dye molecules through the electrolyte solution I - are restored to provide electronic returns to the ground state (3), dye molecules was reborn (4), at the same time - I also was oxidized into $\mathrm{I}^{3-}$; The electrolyte of $\mathrm{I}^{3-}$ spread to the electrode (5), on the electrode surface by the inflow of external electronic back into the I - (6), the excitation - oxidation - reduction of recycling system had a complete steady process of photocurrent (7).

$$
\begin{aligned}
& \mathrm{S}^{*}-\mathrm{e}^{-(\mathrm{CB})} \rightarrow \mathrm{S}^{+} \\
& 3 \mathrm{I}^{-}+2 \mathrm{~S}^{+} \rightarrow \mathrm{I}_{3}^{-} \\
& \mathrm{S}^{+}+\mathrm{e}^{-(\mathrm{CB})} \rightarrow \mathrm{S} \\
& \mathrm{e}^{-(\mathrm{CB})} \rightarrow \mathrm{e}^{-(\mathrm{BC})} \\
& \mathrm{I}_{3}+2 \mathrm{e}^{-(\mathrm{CB})} \rightarrow 3 \mathrm{I}^{-} \\
& \mathrm{I}_{3}^{-}+2 \mathrm{e}^{-(\mathrm{CE})} \rightarrow 3 \mathrm{I}^{-} \\
& \mathrm{S}+\mathrm{hv} \rightarrow \mathrm{S}^{*}
\end{aligned}
$$

\section{The Research Progress of Sensitizing Dyes}

\subsection{Inorganic dyes}

Study more in inorganic dye photosensitizer is synthetic dye pyridine ruthenium system. Although ruthenium Quito pyridine excited states of dyes has a long life, high yield rate of the quantum, the visible light region has the strong absorption capacity and reversible REDOX cycle the advantage such as being strong of performance, but the current multigang pyridine class ruthenium sensitizer in precious metals ruthenium price is higher, and there is pollution to the environment, choose the cheaper metal porphyrins and gold phthalocyanine organic dyes, such as more appropriate.

\subsection{Organic dyes}

Porphyrin class dye cost is low, and the absorption coefficient degree is high, but the porphyrin dyes in the red light district and close to red light district without absorption, under the sunlight wavelength 
limit its further improvement in photoelectric conversion. Phthalocyanine and cyanine dyes because of its stable chemical properties, absorption efficiency is high, thus early on in the application of photosensitive agent. A research report, phthalocyanine organic dyes photoelectric conversion efficiency is about 4\%[4]. But phthalocyanine type dye in more side effects exist in the process of synthesis, process is more complex, so it is difficult to synthesize a single phthalocyanine kind of pure substance.

\subsection{Natural dyes}

Synthetic dyes in the process of production consume large amounts of oil resources, not only produce pollution to the environment, and do great harm to human body health. People tend to be more excellent performance, widely distributed in nature and green natural dyes. Such as plant leaf chlorophyll and anthocyanin of all has the very high photosensitive properties and has the potential value of the research, so that more researchers to explore from the natural dye or pigment in screening for photoelectric conversion and has high absorbance of the dye. Firstmore, natural dyes to improve the efficiency of the dye sensitized solar cell and future industrialization of sensitizing dyes have far-reaching influence; On the other hand natural dye green, environmental protection, renewable but also meet the needs of its market. Therefore natural dye Yu Minhua has important research value and application of solar cells.

\subsection{Completed the study of sensitizing dyes}

Dye-sensitized photoelectrochemical cell dye sensitizer and redox couples have certain requirements, the following conditions must be met as flower: 1)In the semiconductor surface with good adsorption, can quickly reach the adsorption equilibrium, and easy to fall off, the dye molecules on the mother should be easy to nanometer semiconductor surface bonding groups, such as $-\mathrm{COOH},-\mathrm{SO}_{3} \mathrm{H},-\mathrm{PO}_{3} \mathrm{H}_{2}$ like; (2) a sufficient negative excited state redox potential to make semiconductors inject electrons into the conduction band; (3)excited state lifetime is long enough, and having high charge transfer efficiency; (4) to match the solar spectrum, as far as possible to extend the light absorption region to the infrared region; higher (5) oxidation state and an excited state stability, not easily broken; (6) the ground state of the dye sensitizer does not solution redox play a role; (7) redox potential of the semiconductor flat band potential difference between the bigger the better; (8) the process of electron transfer rate constants in the larger, so less energy loss.

In recent years, researchers have started to use density functional theory (DFT) [5, 6] and time-dependent density functional theory (TD DFT) [7, 9] to dihydrogen indoles dyes are studied, the sifting relatively good DSSC photosensitizer. For molecular design, the theoretical calculation method is a powerful tool to calculate the conclusion given synthesis has a guiding significance for the new efficient dye $[10,14]$. Series of DFT calculations dihydrogen indole dye to compute some key chemical bond length of these dyes, in order to compare the stability of several kinds of dye molecules. TD DFT calculation it's under the vacuum ultraviolet - visible absorption spectra, in order to compare the several kinds of dye absorption spectrum and the solar radiation spectrum matching and these dyes as sensitizer of DSSC use of solar energy.

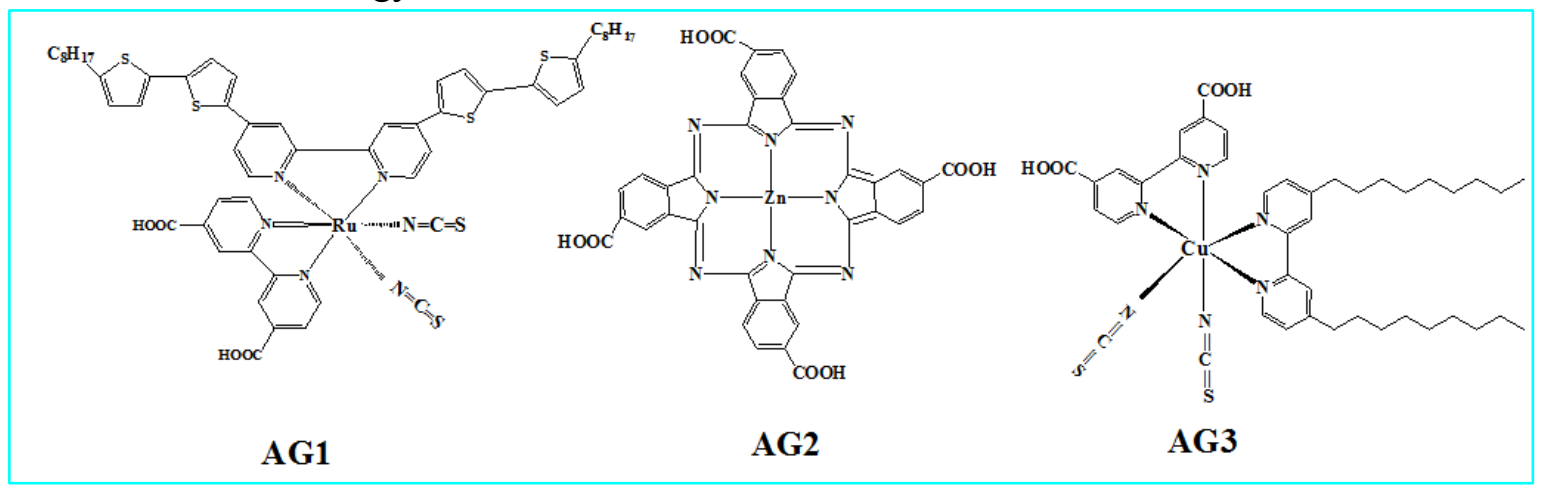

Fig2. The chemical structure of AG1, AG2 and AG3

According to the experimental data obtained by molecular absorption spectra and molecular analysis model changes in solvent in the ground state and the excited state, using the density functional method, molecule at the ground state geometry optimization; through TD-DFT method optimization, in a vacuum 
state changes with the solvent molecules of excitation energies, absorption spectra and vibration intensity, in order to optimize improve ability change in efficiency, by comparing the bond length, frontier molecular orbital energy levels of the molecular orbital's near, excitation energy, absorption spectrum, vibration intensity, the comparison can obtain relative performance in all aspects of the high efficiency, stable quality and dye molecules weather-ability, stability improvement. The structure of the two dyes is typical D-pi-A structure, with the same receptor ((4-(cyanomethyl) benzoic acid groups), and two kinds of dyes with different conjugated spacers (AG1 benzene unit and AG2 Thiophene Units) and different donor unit (AG1 triaromatic amine and AG2 tri aryl donors. AG1 and thiophene ring and benzene ring dihedral angle D C6-C7-C8-S9, D (S9-C10-C11-S12), D (C14-C15-C16-C17) respectively for 22.83129 $160.39960-20.39184$. But for AG2, the degree of distortion of the conjugated bridge of the thiophene ring is lower than that of $\mathrm{AG} 3$, the lower the degree of twist, the more stable the dye molecule, and the longer the service life of DSSC.

\section{Foreground and Prospect}

Solar energy as one of the main energy in the world today, its status is also increasingly brought to the attention of the international. So as the main research direction of the solar cell dye-sensitized cells also arises at the historic moment, one of the natural dye sensitization battery researches began to appear on the international agenda. Natural dyes the project will be for the development, protection and utilization of natural pigments in the region to provide statistical basis. Although many dye molecules have been studied before, but nature, there are many plants and some microbes in natural light conversion efficiency of high or exciting, therefore, can be by nature plant general census, high screening efficiency of plant pigments, can yet be regarded as a kind of ideal solution; By investigating ecological characteristics, and plant pigment extraction, scanning analysis of absorption spectrum, and then using mathematical methods, sort out the types of biophysics characteristics of photoelectric conversion rate is higher for the analysis of the system, the comprehensive study on the acquisition of plant pigments, and the analysis of applied mathematics, natural pigment and stronger ability of electron transfer in pigment, natural dye sensitized cells for the future study lay the foundation

\section{Acknowledgements}

This work has been supported by the key projects in innovation and entrepreneurship of College Students training program, Heilongjiang Province China. №: 201510222015 and Heilongjiang Provincial Department of Education Science and Technology Research Project. №: 12511565.

\section{References}

[1] Grätzel M. Corrigendum to "Conversion of sunlight to electric power by nanocrystalline dye sensitized solar cells [J]. Photochem. Photobiol. A: Chemistry, 2004, 168(3): 235.

[2] O'regan B, Grätzel M. A low-cost, high-efficiency solar cell based on dye-sensitized colloidal $\mathrm{TiO}_{2}$ films [J]. Nature, 1991, 353 (6346): 737-740.

[3] O'regan B, Grätzel M. A low-cost, high-efficiency solar cell based on dye-sensitized colloidal $\mathrm{TiO}_{2}$ films [J]. Nature, 1991, 353(6346): 737-740.

[4] James C, Hideo T. Fabrication of solid-state dye-sensitized $\mathrm{TiO}_{2}$ solar cells combined with polypyrrole [J]. Journal of Applied Phycology, 2000, 12: 207-218.

[5] Hohenberg P, Kohn W. Phys Rev 136: pp [J]. B864, 1964.

[6] Kohn W, Sham L J. Phys Rev 140: pp [J]. A1133, 1965.

[7] Bauernschmitt R, Ahlrichs R. Treatment of electronic excitations within the adiabatic approximation of time dependent density functional theory [J]. Chemical Physics Letters, 1996, 256(4): 454-464. 
[8] Bauernschmitt $\mathrm{R}$, Häser $\mathrm{M}$, Treutler $\mathrm{O}$, et al. Calculation of excitation energies within time-dependent density functional theory using auxiliary basis set expansions [J]. Chemical physics letters, 1997, 264(6): 573-578.

[9] Hirata S, Head-Gordon M. Time-dependent density functional theory for radicals: An improved description of excited states with substantial double excitation character [J]. Chemical physics letters, 1999, 302(5): 375-382.

[10] Hara K, Sato T, Katoh R, et al. Molecular design of coumarin dyes for efficient dye-sensitized solar cells [J]. The Journal of Physical Chemistry B, 2003, 107(2): 597-606.

[11] Kim S, Lee J K, Kang S O, et al. Molecular engineering of organic sensitizers for solar cell applications[J]. Journal of the American Chemical Society, 2006, 128(51): 16701-16707.

[12] Kurashige Y, Nakajima T, Kurashige S, et al. Theoretical investigation of the excited states of coumarin dyes for dye-sensitized solar cells [J]. The Journal of Physical Chemistry A, 2007, 111(25): 5544-5548.

[13] Zhang X, Zhang J J, Xia Y Y. Molecular design of coumarin dyes with high efficiency in dye-sensitized solar cells [J]. Journal of Photochemistry and Photobiology A: Chemistry, 2008, 194(2): 167-172.

[14] Li Y, Liu S, Chen M, et al. Photoinduced intermolecular and intramolecular charge transfer in the mixed coaggregates of pyrazoline and dicyanonaphthalene[J]. Journal of Photochemistry and Photobiology A: Chemistry, 2009, 205(2): 139-144. 